\title{
Effective Field Theory for Long Strings
}

\section{Baker}

Department of Physics, University of Washington, Seattle

Box 351560, Seattle WA 98195, USA

E-mail: mbaker4du.washington.edu

In previous work we used magnetic $\mathrm{SU}(\mathrm{N})$ gauge theory with adjoint representation Higgs scalars to describe the long distance quark-antiquark interaction in pure Yang-Mills theory, and later to obtain an effective string theory. The empirically determined parameters of the non-Abelian effective theory yielded $Z_{N}$ flux tubes resembling those of the Abelian Higgs model with LandauGinzburg parameter equal to $1 / \sqrt{2}$, corresponding to a superconductor on the border between type I and type II. However, the physical significance of the differences between the Abelian and the $Z_{N}$ vortices was not elucidated and no principle was found to fix the value of the 'LandauGinzburg parameter' $\kappa$ of the non-Abelian theory determining the structure of the $Z_{N}$ vortices.

Here we reexamine this point of view. We propose a consistency condition on $Z_{N}$ vortices underlying a confining string. This fixes the value of $\kappa$. The transverse distribution of pressure $p(r)$ in the resulting $Z_{N}$ flux tubes provides a physical picture of these vortices which differs essentially from that of the vortices of the Abelian Higgs model. We speculate that this general picture is valid independent of the details of the effective magnetic gauge theory from which it was obtained. Long wavelength fluctuations of the axis of the $Z_{N}$ vortices lead from an effective field theory to an effective string theory with the Nambu-Goto action. This effective string theory depends on a single parameter, the string tension $\sigma$. In contrast, the effective field theory has a second parameter, the intrinsic width $1 / M$ of the flux tube, and is applicable at intermediate distances in a range between $0.2 \mathrm{fm}$ and $1 \mathrm{fm}$, where the contribution of the intrinsic width increases the flux tube width over that predicted by effective string theory.

Xth Quark Confinement and the Hadron Spectrum

8-12 October 2012

TUM Campus Garching, Munich, Germany 


\section{Introduction}

The principal goal of this talk is to reexamine magnetic $S U(N)$ gauge theory which we have used [1] as an effective field theory of the long distance quark-antiquark interaction, and to elucidate the properties of the $Z_{N}$ flux tubes found in the theory,

In Sections 2 to 4 we write down the Lagrangian of the effective $S U(N)$ gauge theory. We obtain a relation, applicable for any configuration of the Higgs fields, between the string tension $\sigma$ and the transverse distribution of pressure $p(r)$ in the resulting $Z_{N}$ flux tubes. In section 5, using this relation, we impose a constraint on $p(r), \int_{0}^{\infty} r p(r) d r=0$, which we speculate is a necessary condition for a flux tube to behave as a string.

In sections 6 and 7 we consider $S U(3)$, where we have found explicit classical $Z_{3}$ flux tube solutions, and we compare these solutions to those found in the Abelian Higgs model. We plot the pressure distributions $p(r)$ in the $Z_{3}$ flux tube, and describe the physical picture that emerges. The pressure is positive near the axis and at larger distances it is negative. It is natural to associate the boundary between the outside and inside of the string with the point at which the pressure vanishes.

In sections 8 and 9 we show that long wavelength fluctuations of the flux tube axis lead from an effective field theory to an effective string theory with a single parameter, $\sigma$. The contribution of these fluctuations to the flux tube width [2] fixes the value of the short distance cutoff $1 / \Lambda$ of the effective field theory at a value less than the intrinsic width $1 / M$. Thus the theory can resolve distance scales on the order of $1 / M$. Finally, we examine the impact of string fluctuations on the domain of applicability of the effective field theory.

\section{Effective Field Theory}

The Lagrangian $\mathscr{L}_{\text {eff }}$ couples magnetic $S U(N)$ gauge potentials, $C_{\mu}$ to three adjoint representation scalar fields $\phi_{i}$. The gauge coupling constant is $g_{m}$.

$$
\begin{gathered}
\mathscr{L}_{e f f}\left(C_{\mu}, \phi_{i}\right)=2 \operatorname{tr}\left(-\frac{1}{4} G^{\mu v} G_{\mu v}+\frac{1}{2}\left(\mathscr{D}_{\mu} \phi_{i}\right)^{2}\right)-V\left(\phi_{i}\right), \\
G_{\mu v}=\partial_{\mu} C_{v}-\partial_{\nu} C_{\mu}-i g_{m}\left[C_{\mu}, C_{v}\right], \mathscr{D}_{\mu} \phi_{i}=\partial_{\mu} \phi_{i}-i g_{m}\left[C_{\mu}, \phi_{i}\right] .
\end{gathered}
$$

The components of the field tensor $G^{\mu v}$ define color electric and magnetic fields $\vec{E}$ and $\vec{B}$.

$$
E^{k}=\frac{1}{2} \varepsilon_{k l m n} G^{l m}, B^{k}=G^{k 0}
$$

The Higgs potential $V\left(\phi_{i}\right)$ is generated from one loop graphs of $\mathrm{SU}(\mathrm{N})$ gauge theory:[1]

$$
V\left(\phi_{i}\right)=\frac{\mu^{2} N}{4} \sum_{i} 22 \operatorname{tr} \phi_{i}^{2}+\frac{4 N \lambda}{3}\left(\operatorname{tr}\left(\sum_{i j} \phi_{i}^{2} \phi_{j}^{2}\right)+\frac{1}{N}\left(\operatorname{tr}\left(\sum_{i} \phi_{i}^{2}\right)\right)^{2}+\frac{2}{N} \sum_{i j}\left(\operatorname{tr} \phi_{i} \phi_{j}\right)^{2}\right),
$$

where the parameter $\mu^{2}$ has dimensions of mass squared and $\lambda$ is dimensionless. The $\mathrm{SU}(\mathrm{N})$ gauge symmetry of $\mathscr{L}_{\text {eff }}$ reflects that of the original $\mathrm{SU}(\mathrm{N})$ Yang-Mills theory.

In the confining vacuum the magnetic $\mathrm{SU}(\mathrm{N}) / Z_{N}$ gauge symmetry is completely broken by a Higgs condensate $<\phi_{i}>=\phi_{i 0}=\phi_{0} J_{i}$, where the three matrices $J_{i}$ are the generators of $\mathrm{N}$-dimensional irreducible representation of the three-dimensional rotation group. The Higgs potential has an absolute minimum at $\phi_{i}=\phi_{i 0}: \phi_{0}^{2}=-\frac{9 \mu^{2}}{8\left(N^{2}-1\right) \lambda}$. 


\section{3. $Z_{N}$ Electric Flux Tubes}

At large distances $r$ from the flux tube axis $\phi_{i}$ and $C_{\mu}$ are a gauge transformation $\Omega(\theta)$ of the vacuum $\phi_{i}=\phi_{i 0}, C_{\mu}=0$, which we can choose to be Abelian; $\Omega(\theta)=\exp (i \theta Y)$.

$$
\phi_{i} \rightarrow \Omega^{-1}(\theta) \phi_{i 0} \Omega(\theta), C_{\mu} \rightarrow \frac{i}{g_{m}} \Omega^{-1}(\theta) \partial_{\mu} \Omega(\theta) .
$$

The requirement that $\phi_{i}$ be single valued $\rightarrow \exp (i 2 \pi Y)$ is an element of $Z_{N}$.

As $r \rightarrow \infty$

$$
\vec{C} \rightarrow \frac{1}{g_{m}, r} \hat{e}_{\theta} Y, \quad \exp \left(i g_{m} \oint \vec{C} \cdot d \vec{l}\right) \rightarrow \exp \left(2 \pi i \frac{k}{N}\right), k=1,2, N-1
$$

Assuming the gauge potential $\vec{C}=C(r) \hat{e}_{\theta} Y$ everywhere implies that the electric field

$$
\vec{E}=-\nabla \times \vec{C}(\vec{x}) Y=-\frac{1}{r} \frac{d(r C(r))}{d r} \hat{e}_{z} Y .
$$

The finiteness of the flux tube energy $\rightarrow \phi_{i}=0$ on the flux tube axis.

\section{Relation Between String Tension and Stress Tensor in SU(N) Flux Tubes}

Using the Abelian ansatz (3.3) and the resulting classical static equation

$$
\nabla \times \vec{E}=-\vec{j}=i g_{m}\left[\phi_{i}, \vec{D} \phi_{i}\right]
$$

to evaluate $\mathscr{L}_{\text {eff }}$ gives the following general relation between the string tension $\sigma$, the stress tensor component $T_{\theta \theta}$, and $\vec{E}(r=0)$, the color electric field on the axis of the flux tube:

$$
\int_{0}^{\infty} 2 \pi r \frac{T_{\theta \theta}(r)}{r^{2}} d r=-2 \operatorname{tr}\left(\frac{2 \pi}{g_{m}} Y \hat{e} \cdot \vec{E}(r=0)\right)-\sigma .
$$

valid for any configuration of the Higgs fields $\phi_{i}$. The quantity $-2 \operatorname{tr}\left(\frac{2 \pi}{g_{m}} Y \hat{e}_{z} \cdot \vec{E}(r=0)\right) R=W$, the work necessary to separate a $q \bar{q}$ pair lying on the z-axis by a distance $R$.

If $T_{\theta \theta}>0$ the gauge repulsion exceeds the Higgs attraction produced by the circulating magnetic currents generated by the Higgs condensate, and (4.2) implies that $W>\sigma R$. That is, when there is net repulsion, the work $W$ needed to separate the $q \bar{q}$ pair a distance $R$ in the fixed final vortex field $\vec{E}(r=0)$ is greater than $\sigma R$, which itself is equal to the work done in a field $\vec{E}$ that is being built up as the $q \bar{q}$ pair is separated.

If there is compensation between the net attractive and repulsive contributions to the pressure $p(r)=T_{\theta \theta} / r^{2}$ averaged over the width of the flux tube; that is, if

$$
\int_{0}^{\infty} 2 \pi r \frac{T_{\theta \theta}}{r^{2}} d r=\int_{0}^{\infty} 2 \pi r p(r) d r=0
$$

then the string tension $\sigma=W / R$, determined by the field $\vec{E}(r=0)$ on the flux tube axis. 


\section{Speculation on Effective Field Theories Describing Long Strings}

Consider now a flux tube connecting a $q \bar{q}$ pair located at $z= \pm R / 2$ having energy $V_{0}(R)$, the heavy quark potential. The force acting on the quarks is determined by the color field at the positions of the quarks and is equal to $d V_{0} / d R$. If the long distance potential $V_{0}(R)=\sigma R$ persists to distances $R$, then this field is fixed by the string tension $\sigma$.

If condition (4.3) is met, the field $\vec{E}$ on the $z$ axis near the midpoint of the flux tube is also fixed by the value of $\sigma$. In this situation, it is consistent to assume the field has the same value, proportional to $\sigma$, at all points on the $z$ axis between the $q \bar{q}$ pair ; that is, the flux tube behaves like a string, consistent with the assumption that the long distance $q \bar{q}$ interaction persists to short distances. This argument fails if condition (4.3) is not satisfied.

We assume that (4.3) must be satisfied for any effective field theory describing the confining string in $\mathrm{SU}(\mathrm{N})$ Yang-Mills theory, and we impose this condition to constrain the parameters in $\mathscr{L}_{\text {eff }}$.

\section{Classical Static SU(3) Flux Tube Solutions}

For SU(3) we have found explicit classical static solutions [1] with

$$
\begin{aligned}
J_{x} & =\lambda_{7}, J_{y}=-\lambda_{5}, J_{z}=\lambda_{2}, Y=\frac{\lambda_{8}}{\sqrt{3}}, \\
\phi_{1} & =\phi_{1}(\vec{x}) \frac{\left(\lambda_{7}-i \lambda_{6}\right)}{2}+\phi_{1}^{*}(\vec{x}) \frac{\left(\lambda_{7}+i \lambda_{6}\right)}{2}, \\
\phi_{2} & =\phi_{2}(\vec{x}) \frac{\left(-\lambda_{5}-i \lambda_{4}\right)}{2}+\phi_{2}^{*}(\vec{x}) \frac{\left(-\lambda_{5}+i \lambda_{4}\right)}{2}, \\
\phi_{3} & =\phi_{3}(\vec{x}) \lambda_{2}, \\
\vec{C} & =C(r) \hat{e}_{\theta} Y, \phi_{1}(\vec{x})=\phi(r) \exp (-i \theta), \phi_{2}(\vec{x})=\phi(r) \exp (i \theta), \phi_{3}(\vec{x})=\phi_{3}(r) .
\end{aligned}
$$

The commutation relations

$$
\left[Y, \lambda_{7}-i \lambda_{6}\right]=\lambda_{7}-i \lambda_{6},\left[Y,-\lambda_{5}-i \lambda_{4}\right]=-\left(-\lambda_{5}-i \lambda_{4}\right),\left[Y, \lambda_{2}\right]=0
$$

show that the Higgs fields $\phi_{1} \phi_{2}, \phi_{3}$ carry $Y$ charge $-1,1,0$ respectively, and that the ansatz (6.1) is consistent with equation (4.1).

We rescale the fields choosing the flux tube radius $\frac{1}{M}$ as the scale of length, making the replacement $r \rightarrow r / M, C \rightarrow \frac{M C}{g_{m}}, \phi \rightarrow \phi_{0} \phi, \phi_{3} \rightarrow \phi_{0} \phi_{3}$, with $M=\sqrt{6} g_{m} \phi_{0}$. Inserting (6.1) into the effective Lagrangian (2.1) yields the energy density $T_{00}$ and the stress tensor component $T_{\theta \theta}$ :

$$
\begin{aligned}
& T_{00}=\frac{4}{3} \frac{M^{2}}{g_{m}^{2}}\left[\frac{1}{2}\left(\frac{1}{r} \frac{d(r C)}{d r}\right)^{2}+\frac{1}{2}\left(C-\frac{1}{r}\right)^{2} \phi^{2}+\frac{1}{2}\left(\frac{d \phi}{d r}\right)^{2}+\frac{1}{4}\left(\frac{d \phi_{3}}{d r}\right)^{2}+V\left(\phi, \phi_{3}\right)\right] \\
& \frac{T_{\theta \theta}}{r^{2}}=\frac{4}{3} \frac{M^{4}}{g_{m}^{2}}\left[\frac{1}{2}\left(\frac{1}{r} \frac{d(r C)}{d r}\right)^{2}+\frac{1}{2}\left(C-\frac{1}{r}\right)^{2} \phi^{2}-\frac{1}{2}\left(\frac{d \phi}{d r}\right)^{2}-\frac{1}{4}\left(\frac{d \phi_{3}}{d r}\right)^{2}-V\left(\phi, \phi_{3}\right)\right]
\end{aligned}
$$


where

$$
V\left(\phi, \phi_{3}\right)=\kappa^{2}\left(\frac{\left(\phi^{2}-1\right)^{2}}{4}+9 \frac{\left(\phi_{3}^{2}-1\right)^{2}}{100}-7 \frac{\left(\phi_{3}^{2}-1\right)\left(1-\phi^{2}\right)}{50}\right), \kappa^{2} \equiv \frac{25}{9} \frac{\lambda}{g_{m}^{2}} .
$$

Separating the gauge contribution and the Higgs contribution to $T_{00}$ and $T_{\theta \theta}$ gives

$$
\begin{aligned}
\int_{0}^{\infty} 2 \pi r T_{00} d r=\sigma=\frac{4}{3} \frac{M^{2}}{g_{m}^{2}} \sigma(\kappa) & =\frac{4}{3} \frac{M^{2}}{g_{m}^{2}}\left(\sigma_{g}(\kappa)+\sigma_{h}(\kappa)\right), \\
\int_{0}^{\infty} 2 \pi r \frac{T_{\theta \theta}}{r^{2}} d r & =\frac{4}{3} \frac{M^{2}}{g_{m}^{2}}\left(\sigma_{g}(\kappa)-\sigma_{h}(\kappa)\right),
\end{aligned}
$$

where

$$
\sigma_{g}(\kappa) \equiv \int_{0}^{\infty} 2 \pi r d r\left(\frac{1}{2}\left(\frac{1}{r} \frac{d(r C)}{d r}\right)^{2}+\frac{1}{2}\left(C-\frac{1}{r}\right)^{2} \phi^{2}\right)
$$

and

$$
\sigma_{h}(\kappa) \equiv \int_{0}^{\infty} 2 \pi r d r\left(\frac{1}{2}\left(\frac{d \phi}{d r}\right)^{2}+\frac{1}{4}\left(\frac{d \phi_{3}}{d r}\right)^{2}+V\left(\phi, \phi_{3}\right)\right)
$$

\section{Results for SU(3) String Tension and Stress Tensor}

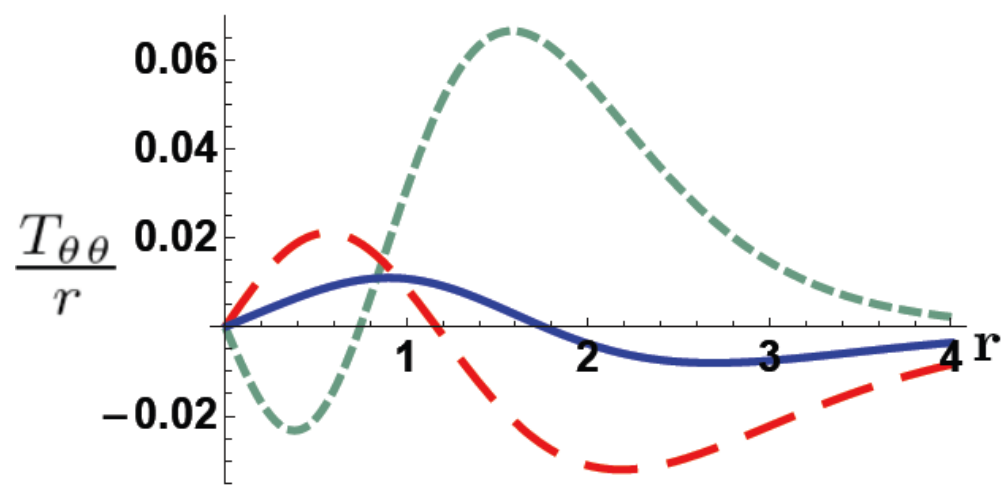

Figure 1: $T_{\theta \theta} / r$ vs $r$. Red, long dashed, $\kappa^{2}=0.5 ; \quad$ blue, thick, $\kappa^{2}=0.6 ; \quad$ green, short dashed, $\kappa^{2}=0.8$

Note that if $\phi_{3}(r)$ has its vacuum value $\phi_{3}=1, V\left(\phi, \phi_{3}\right)$ reduces to the Higgs potential of the Abelian Higgs model with Landau Ginzburg parameter $\kappa$. Furthermore, numerical solution of the classical equations shows that $\phi<1$ and $\phi_{3}>1$ everywhere; hence the term coupling $\phi$ and $\phi_{3}$ in (6.5) is attractive. This additional attraction in $V\left(\phi, \phi_{3}\right)$ reduces the energy of the $Z_{3}$ vortex below that of the Abrikosov-Nielsen-Olesen vortex of the Abelian Higgs model. The ANO vortex can then be viewed as an unstable configuration of the non-Abelian theory that subsequently decays to the stationary classical solution $\phi_{3}(r)$.

Conditions (4.3) and (6.7) yield $\sigma_{g}(\kappa)=\sigma_{h}(\kappa)$, which determines the physical value of $\kappa ; \kappa^{2} \sim 0.6$. The string tension $\sigma\left(\kappa^{2} \approx 0.6\right) \approx 3.1$, approximately equal to its value in the Abelian Higgs model at $\kappa^{2}=1 / 2$. Figure 1 shows $T_{\theta \theta} / r$ evaluated at the classical solution 
as a function of $r$ for three values of $\kappa^{2}$. For $\kappa^{2} \approx 0.6$, where condition (4.3) is satisfied, $T_{\theta \theta}=0$ at $r \equiv r * \sim 1.7 / M$; there is repulsion at $r \leq r *$ and attraction at $r>r *$. It is natural to identify $r *$ as a boundary, separating the inside of the flux tube from its exterior.

In contrast, in the Abelian Higgs model $\kappa=\frac{1}{\sqrt{2}}$ is a BPS state [3], and condition (4.3) is satisfied exactly because the components $T_{\theta \theta}$ and $T_{r r}$ of the stress tensor vanish for all $r$ [4], and thus the profile of $T_{\theta \theta}(r)$ does not reveal the boundary of the flux tube.

We speculate that the difference between the properties of the stress tensor inside (positive net pressure) and outside the flux tube (negative net pressure) is a fundamental physical property of flux tubes giving rise to a confining string. The difference between the Abelian and non-Abelian theories is caused by the additional attractive interaction between the scalar particles, which breaks the supersymmetry [5] giving rise to the BPS Abelian Higgs vortex, and stabilizes the non- Abelian flux tube. Indeed, as we have seen, the additional attraction in the Higgs potential of the non-Abelian theory is approximately balanced at $\kappa^{2} \approx 0.6$ by the additional repulsion associated with the fact that $\kappa^{2}>1 / 2$.

\section{From Effective Field Theory to Effective String Theory}

The Higgs fields $\phi$ vanish on the axis $L$ of the static flux tube. Long wavelength fluctuations of the axis $L$ of a flux tube connecting a quark-antiquark pair sweep out a space time surface $\tilde{x}^{\mu}(\zeta)$ on which $\phi$ vanishes. The Wilson loop $W(\Gamma)$ of Yang-Mills theory is the path integral over all field configurations for which the Higgs fields vanish on some surface $\tilde{x}^{\mu}(\zeta)$ whose boundary is the loop $\Gamma$.

$$
W(\Gamma)=\int \mathscr{D} C_{\mu} \mathscr{D} \phi \exp \left(i S\left(C_{\mu}, \phi\right), \quad S\left(C_{\mu}, \phi\right)=\int d x \mathscr{L}_{e f f}\left(C_{\mu}, \phi\right) .\right.
$$

We transform $W(\Gamma)$ to a path integral over the vortex sheets $\tilde{x}^{\mu}(\zeta)$ in two stages:

1. We fix the location $\tilde{x}^{\mu}(\zeta)$ of the vortex and integrate over field configurations $C_{\mu}(x), \phi(x)$ for which $\left.\phi(x)\right|_{x=\tilde{x}(\zeta)}=0$. The integration (8.1) over these configurations $\rightarrow S_{\text {eff }}(\tilde{x})$, the action of the effective string theory.

2. We then integrate over all surfaces $\tilde{x}^{\mu}(\zeta)$. This integration puts $W(\Gamma)$ into the form of a partition function of an effective string theory: [6]

$$
W(\Gamma)=\int \mathscr{D} \tilde{x}^{\mu} \ldots \exp \left[i S_{e f f}\left(\tilde{x}^{\mu}\right)\right]
$$

The path integral (8.2) goes over the two transverse fluctuations of the world sheet $\tilde{x}^{\mu}(\zeta)$.

The field modes contributing to $S_{\text {eff }}\left[\tilde{x}^{\mu}\right]$ have masses $>M$. Fluctuations of wavelength $>1 / M$ are string fluctuations accounted for by (8.2). We can then replace integrations (8.1) over field configurations $C_{\mu}, \phi$ by the classical configuration minimizing $S\left(C_{\mu}, \phi\right)$ for fixed position $x^{\mu}(\zeta)$ of the vortex.

$$
\exp \left(i S_{\text {eff }}\left(\tilde{x}^{\mu}(\zeta)\right)\right) \approx \exp \left(i S^{\text {class }}\left(\tilde{x}^{\mu}(\zeta)\right)\right) .
$$

When condition (4.3) is satisfied, the linear potential persists when a straight flux tube is shortened. Likewise, bending the flux tube slightly gives a change in energy proportional 
to the change $\Delta R$ in length: $\Delta E=\sigma \Delta R$. The action of the effective of the effective field theory becomes the Nambu-Goto action proportional to the area of the vortex sheet.

$$
S_{e f f}\left(\tilde{x}^{\mu}\right)=\sigma \int d^{2} \xi \sqrt{-g(\xi)} \equiv S_{N G}\left(\tilde{x}^{\mu}\right) .
$$

\section{Heavy Quark Potentials and Flux Tube Shape}

To obtain the heavy quark potential $V_{0}(R)$ and transverse energy profiles between static quarks separated by distance $R$ we couple the vector potential $\vec{C}$ to a Dirac string, writing

$$
\vec{E}=-\nabla \times \vec{C}-\frac{2 \pi}{g_{m}} \delta(x) \delta(y)(\theta(z+R / 2)-\theta(z-R / 2)) \hat{e}_{z} Y
$$

in the Lagrangian $\mathscr{L}_{\text {eff }}$, and solving the resulting static equations [7]. We compared the results [8] with lattice data for heavy quark potentials [9], and found that $g_{m} \approx 3.91$; i. e., $M \approx 1.9 \sqrt{\sigma}$. Furthermore, these calculations were consistent with $\mathrm{SU}(2)$ lattice simulations [10] for transverse energy profiles for a range of interquark spacings $0.25 / \sqrt{\sigma} \leq R<2 / \sqrt{\sigma}$.

The above calculations did not explicitly include the contribution of string fluctuations. However, string fluctuations renormalize the intrinsic width and therefore they are to some extent accounted for in the empirically determined value of $M$. For distances larger than $\sim 1 / \sqrt{\sigma}$, string fluctuations become dominant, leading to the logarithmic increase of the mean square width of the flux tube at its midpoint [11];

$$
w^{2}(R / 2)=\frac{d-2}{2 \pi \sigma} \log \frac{R}{r_{0}} .
$$

Recent lattice simulations of $(2+1) d S U(2)$ Yang-Mills theory [2] extending to distances $R=36 / \sqrt{\sigma}$ gave excellent agreement with the predictions of effective string theory for distances $R>1.5 / \sqrt{\sigma}$, and determined the value of $r_{0}=0.364 / \sqrt{\sigma}$. (Interpreting $1 / r_{0}=\Lambda$ as the cutoff of the effective field theory gives $\Lambda \approx 2.75 \sqrt{\sigma} \approx 1.41 M$.) However, for distances $1.5 / \sqrt{\sigma}>R>0.2 / \sqrt{\sigma}$ the lattice simulations of $w^{2}(R / 2)$ lie above the prediction (9.2), indicating that the intrinsic width of the flux tube must be taken into account at these $q \bar{q}$ separations. It is in this intermediate range, shown schematically in Figure 2, that we can test the physical picture of the confining string given by the effective field theory.

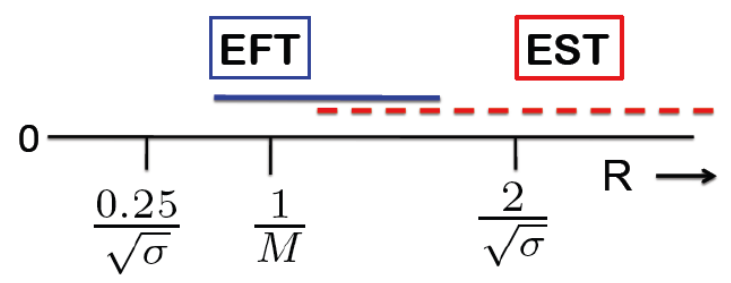

Figure 2: Schematic showing approximate domains of applicability of Effective Field Theory (EFT) (solid blue line) and Effective String Theory (EST) (red dashed line). 
With use of analytic regularization [12], string fluctuations do not renormalize the string tension $\sigma$, and hence its physical interpretation as the energy per unit length of the classical flux tube is preserved. The leading large distance correction to the heavy quark potential is the Lüscher term $-\pi(d-2) / 24 R$ [13], which can be regarded as a renormalization of the intrinsic width at the intermediate distances shown as the region of overlap in Figure 2.

\section{Summary and Future Work}

We have presented a physical picture of the $Z_{N}$ flux tubes giving rise to a confining string. In this picture net positive pressure in the interior of the $Z_{N}$ vortices balances net negative pressure outside. (Perhaps at the deconfinement temperature the flux tube 'bursts'! ) We speculate that this general description is valid, independent of the details of the effective magnetic gauge theory from which it was obtained.

Comparison with lattice simulations at intermediate distances would test our hypothesis that there is an effective field theory underlying the confining string. Since $M \sim 3$ times $T_{C}$, the SU(3) deconfinement temperature, the theory should be applicable for a range of temperatures in the deconfined phase, where it was used [14] in a preliminary study of spatial Wilson loops and where we expect some manifestation of the Higgs field.

\section{Acknowledgment:}

I would like to thank the organizers for the opportunity to participate in this very stimulating conference.

\section{References}

[1] M. Baker, J. S. Ball and F. Zachariasen, Phys. Rev. D41, 2612 (1990).

[2] F. Gliozzi, M. Pepe and O.-J Weise, Phys. Rev. Lett. 104, 232001 (2010).

[3] E. B. Bogomolny, Sov. J. Nucl. Phys. 24, 449 (1976) [Yad. Fiz. 24, 861 (1976)]; M. K. Prasad and C. M. Sommerfield, Phys. Rev. Lett. 35, 760 (1975).

[4] H. J. de Vega and F. A. Schaposnik, Phys. Rev. D14, 1100 (1976).

[5] P. Fayet, Il Nuovo Cimento 31A, 626 (1976).

[6] M. Baker and R. Steinke, Phys. Rev. D63, 094013 (2001); D65, 114042 (2002).

[7] M. Baker, J. S. Ball and F. Zachariasen, Phys. Rev. D44, 3328 (1991).

[8] M. Baker, J. S. Ball and F. Zachariasen, Phys. Rev. D56, 4400 (1997).

[9] G. S. Bali, K. Schilling and A. Wachter. Phys. Rev. D 562566 (1997).

[10] A. M. Green, C.Michael and P. S. Spencer, Phys. Rev. D55, 1216 (1997).

[11] M. Lüscher, G. Münster and P. Weisz, Nucl. Phys. B180, 1 (1981).

[12] K. Dietz and T. Filk, Phys. Rev. D27, 2944 (1982).

[13] M. Lüscher, Nucl. Phys B180, 317 (1981).

[14] M. Baker, Phys. Rev. D 78, 014009, (2008). 